J. D. Badia, L. Monreal, V. Sáenz de Juano-Arbona A. Ribes-Greus. Dielectric spectroscopy of recycled polylactide. Polymer Degradation and Stability 2014; 107, 21-27. ISSN 0141-3910, http://dx.doi.org/10.1016/j.polymdegradstab.2014.04.023.

\title{
DIELECTRIC SPECTROSCOPY OF RECYCLED POLYLACTIDE
}

\section{J. D. Badiaa ${ }^{1,2}$, L. Monreal ${ }^{3}$, V. Sáenz de Juano-Arbona ${ }^{1}$, A. Ribes-Greus ${ }^{1, *}$}

This is an open-access version, according to http://www.sherpa.ac.uk/romeo/issn/0141-3910/

Full text available at http://www.sciencedirect.com/science/article/pii/S0141391014001785

DOI: dx.doi.org/10.1016/j.polymdegradstab.2014.04.023

Please, cite it as:

J. D. Badia, L. Monreal, V. Sáenz de Juano-Arbona A. Ribes-Greus. Dielectric spectroscopy of recycled polylactide. Polymer Degradation and Stability 2014; 107, 21-27

${ }^{1}$ Instituto de Tecnología de los Materiales (ITM),

Universidad Politècnica de València (UPV)

Camino de Vera S/N, 46022 Valencia, Spain.

${ }^{2}$ Departamentd'Enginyeria Química.

Escola Tècnica Superior d'Enginyeria. Universitat de València.

Av. de la Universitat, s/n, 46100, Burjassot, Spain.

${ }^{3}$ Centro de Investigación en Tecnologías Gráficas,

Universitat Politècnica de València

Camino de Vera S/N, 46022 Valencia, Spain.

*Corresponding author: A. Ribes-Greus aribes@ @ter.upv.es 


\title{
DIELECTRIC SPECTROSCOPY OF RECYCLED POLYLACTIDE
}

\author{
J.D. Badia ${ }^{1,2}$, L. Monreal ${ }^{3}$, V. Sáenz de Juano-Arbona ${ }^{1}$, A. Ribes-Greus ${ }^{1, *}$ \\ ${ }^{1}$ Instituto de Tecnología de Materiales (ITM), \\ Universitat Politècnica de València \\ Camino de Vera s/n, E-46022 Valencia, Spain \\ ${ }^{2}$ Departament d’ Enginyeria Química, \\ Escola Tècnica Superior d' Enginyeria \\ Universitat de València \\ Avinguda de les Universitats s/n, E-46100 Burjassot, Spain \\ ${ }^{3}$ Centro de Investigación en Tecnologias Gráficas, \\ Universitat Politècnica de València \\ Camino de Vera s/n, E-46022 Valencia, Spain \\ *corresponding author: A. Ribes-Greus \\ aribes@ter.upv.es
}

Keywords: recycling, thermo-mechanical degradation, poly(lactide) (PLA), dielectric thermal analysis (DETA), segmental dynamics, dynamic fragility

\begin{abstract}
:
The effects of multiple mechanical recycling on amorphous polylactide (PLA) were simulated by means of five successive injection-grinding cycles. The influence of the induced thermo-mechanical degradation on the dielectric properties of PLA was analysed. The relaxation spectra were studied in terms of the complex dielectric permittivity ( $\varepsilon^{\prime}$ and $\left.\varepsilon^{\prime \prime}\right)$ and the dielectric loss tangent, $\operatorname{tg}(\delta)$ in the frequency range from $10^{-2}$ to $10^{7} \mathrm{~Hz}$ over the temperature interval from $0^{\circ} \mathrm{C}$ to $140^{\circ} \mathrm{C}$. It was possible to distinguish two relaxations zones, one at low temperatures and high frequencies ( $\beta$ relaxation) and another at higher temperatures and lower frequencies ( $\alpha$ relaxation). The individual relaxations were characterised using the Havriliak-Negami model and the effect of conductivity at high temperatures was subtracted. The thermal activation of the $\beta$ relaxations were explained on an Arrhenius model basis while the $\alpha$ relaxation was modelled using Vogel-Fulcher-Tammann-Hesse (VFTH) equations. The study of the segmental dynamics showed a decrease in the fragility parameters, connected with the decrease of molar mass, along with an increase in the free volume, the higher the number of reprocessing cycles were applied, particularly up to the second reprocessing step.
\end{abstract}




\section{Introduction}

Polylactides have numerous interesting properties and gained much attention [1-3] but the increase of this new source of polymeric waste would have to be managed in a near future. Among all material recovery methods [4], mechanical recycling represents one of the most successful processes, although during reprocessing, polymers are subjected to the influence of several degrading agents that may modify the structure [5] and consequently change their long-term properties [6-11].

Previous studies showed the simulation of mechanical recycling by multiple processing to assess the effects of thermo-mechanical degradation on polylactide [12-16], by means of Matrix-Assisted Laser Desorption/Ionization Time-of-Flight Mass Spectrometry MALDI-TOF-MS, Scanning Electron Microscopy SEM, Tensile and Impact testing, Differential Scanning Calorimetry DSC, Fourier-Transform Infrared Spectroscopy FT$I R$, Viscosimetry and Dynamical-Mechanical Thermal Analysis DMTA. Shortly, although results showed that there were non-significant changes in the nature of the functional groups of PLA, there was a remarkable reduction in molar mass due to chain scission, which was subscribed by characterisation of the cold-crystallization phenomenon found during DSC and DMTA. The effect of chain shortening studied on the glass-rubber relaxation showed an increase in free-volume affecting the segmental dynamics of PLA.

Dielectric spectroscopy is a very useful tool to study the structure and the dynamics of polymer segments measured as the response of polylactide in each one of the recycled processes to an electrical perturbation field over a wide frequency range at different temperatures. Thus the purpose of this work was to extent the characterization of the influence of multiple processing on PLA microstructure and segmental dynamics and to relate it to previous results. 


\section{Experimental procedure and calculations}

\subsection{Materials and reprocessing simulation}

Polylactide (PLA) 2002D was a thermo-forming grade PLA obtained from Natureworks LLC (Minnetonka, MN) as pellets, Prior to processing, virgin PLA pellets were dried during $2 \mathrm{~h}$ at $80^{\circ} \mathrm{C}$ in a dehumidifier Conair Micro-D FCO 1500/3 (UK), in order to remove as much humidity as possible. Afterwards, the samples were processed by means of injection moulding in an Arburg 420 C 1000-350 (Germany) single-screw model (diameter $\Phi=35 \mathrm{~mm}$, length $/ \Phi=23$ ) injector. The temperature gradient set from hopper to nozzle was $160,170,190,200$ and $190^{\circ} \mathrm{C}$, whereas the moulds were set at 15 ${ }^{\circ} \mathrm{C}$. Cooling time residence was ca. $40 \mathrm{~s}$ and total residence time ca. 60s. Samples were dried before each processing cycle. After injection, a fraction of the samples was kept as test specimens and the rest was ground by means of a cutting mill Retsch SM2000 (UK), which provided pellets of size $d<20 \mathrm{~mm}$ to be fed back into the process. Up to five processing cycles were applied under the same conditions to obtain the different testing specimens of reprocessed PLA (RPLA-i, with i: 1-5). Afterwards, $1 \mathrm{~mm}$ thick prismatic probes were obtained by compression moulding, as described elsewhere [17].

\subsection{Dielectric Spectroscopy}

The dielectric thermal analysis (DETA) of the recycled polylactides were obtained using an alpha mainframe frequency analyser in conjunction with an active cell (Concept 40, Novocontrol Technologies BmgH \& Co. Kc, Hundsangen, Germany). The response was measured in the frequency range $f=10^{-2}-10^{7} \mathrm{~Hz}$, at temperatures between $0^{\circ} \mathrm{C}$ and $140{ }^{\circ} \mathrm{C}$, controlled by the Quatro system (Novocontrol Technologies BmgH \& Co. Kc, Hundsangen, Germany). The spectra were obtained under isothermal conditions, by increasing steps of $5{ }^{\circ} \mathrm{C}$. The sample electrode assembly (SEA) consisted in two stainless steel electrodes filled with the polymer. The diameters of the electrodes were $20 \mathrm{~mm}$ and the thickness was kept around $300 \mu \mathrm{m}$.

Several variables were considered in the study of the dielectric behaviour of the recycled polylactides. The complex dielectric permeability, $\varepsilon^{*}$, the real $\varepsilon^{\prime}$ and imaginary $\varepsilon$ ' parts of the dielectric permittivity, as well as $\operatorname{tg}(\delta)$ were analysed, according to equations 1 and 2.

$$
\begin{gathered}
\mathcal{E}^{*}=\varepsilon^{\prime}-i \varepsilon^{\prime \prime}(1) \\
\tan (\delta)=\frac{\varepsilon^{\prime \prime}}{\varepsilon^{\prime}}
\end{gathered}
$$




$$
\tan (\delta)=\frac{\varepsilon^{\prime \prime}}{\varepsilon^{\prime}}
$$

At temperatures above the glass transition $T_{G}$, the ohmic conduction due to charge carriers, $\sigma^{*}(E q .3)$, frequently dominates the loss contribution $\left(\varepsilon^{\prime \prime}\right)$, potentially masking dielectric relaxations, and was therefore taken into account.

$$
\sigma^{*}=\varepsilon^{*} \cdot e_{0} \cdot \omega=\sigma^{\prime}+i \sigma^{\prime \prime}(3)
$$

At sufficiently high temperatures and low frequencies, the values of $\varepsilon$ "' and $\operatorname{tg}(\delta)$ rose exceeding the typical range of dielectric relaxations, suggesting the prevalence of conductivity in that region. Hence the conduction-free dielectric loss $\varepsilon^{\prime \prime}{ }_{N C}$ was determined by Eq. 4 [18].

$$
\varepsilon^{\prime \prime}{ }_{N C}=\varepsilon^{\prime \prime}-\frac{\sigma_{0}}{\varepsilon_{0} 2 \pi f^{S}}(4)
$$

with $f$ the frequency in Hertz $(\mathrm{Hz}), \sigma_{0}$ a pre-exponential coefficient, and an exponent, $S$, normally close to 1 , and subtracted from the dielectric response. Data treatment is not shown for the sake of conciseness.

The $\varepsilon^{*}$ curves were modeled by an Havriliak-Negami (HN) function (Eq. 5) [19-20]

$$
\varepsilon^{*}-\varepsilon_{\infty}=\sum_{k}\left[\frac{\Delta \varepsilon}{\left\{1+\left(i w \tau_{H N k}\right)^{\alpha_{k}}\right\}^{\beta_{k}}}\right]
$$

, where $\alpha_{k}$ and $\beta_{k}$ are parameters corresponding to the width and asymmetry of the relaxation time distributions, respectively; $\tau_{H N}$ is the Havriliak-Negami relaxation time and $\Delta \varepsilon=\varepsilon_{S}-\varepsilon_{\infty}$ the dielectric strength (with $\varepsilon_{S}$ and $\varepsilon_{\infty}$ are the real part of the permittivity when the angular frequencies are $\omega \rightarrow 0$ and $\omega \rightarrow \infty$, respectively). The sub index $k$ represents the number of the individual HN contributions, if any. Finally, the relaxation times and frequencies $\left(f_{\max }=1 / \tau_{\max }\right)$ of the HN individual $\varepsilon^{*}$ curves were calculated according to Eq.6 [19]

$$
\tau_{\max }=\tau_{H N}\left[\frac{\sin \left(\frac{\pi\left(\alpha_{N H}\right) \beta_{N H}}{2\left(\beta_{N H}+1\right)}\right)}{\sin \left(\frac{\pi\left(\alpha_{N H}\right)}{2\left(\beta_{N H}+1\right)}\right)}\right]^{1 / \alpha_{N H}}
$$

In addition, the thermal activation of the dielectric phenomena was characterised in Arrhenius maps using the maximum frequency of the relaxations at each isotherm,

$$
f_{\max }=f_{0} \exp \left(\frac{-E a}{R \cdot T}\right)
$$


described by either linear Arrhenius (Eq. 7) or Vogel-Fulcher-Tammann-Hesse (VTFH, Eq. 8) models [21-24]

$$
\begin{array}{r}
f_{\max }=f_{0} \exp \left(\frac{-E a}{R \cdot T}\right) \\
\tau(T)=\tau_{0} \cdot \exp \left(\frac{B}{T-T_{V F T H}}\right)=\tau_{0} \cdot \exp \left(\frac{D \cdot T_{V F T H}}{T-T_{V F T H}}\right)
\end{array}
$$

,where $E a$ is the apparent activation energy, $f_{0}$ is a pre-exponential term, $\tau$ is the relaxation time (s), that is $(2 \cdot \pi \cdot f)^{-1}$, and $f$ is the linear frequency of the DETA tests, $\tau_{0}$ is a time reference scale, and $B(\mathrm{~K})$ and $T_{V F T H}(\mathrm{~K})$ are positive parameters specific to the material. $T_{V F T H}$ typically appears $40-60 \mathrm{~K}$ below the glass transition temperature $T_{G}$. It is common to rewrite the parameter $B$ into $B=D \cdot T_{V F T H}$, where $D$ is a no dimensional factor termed as fragility or strength parameter. Qualitatively, $D$ is related to the topology of the theoretical potential energy surface of the system, where fragile systems $(D \leq 6)$ present high density of energy minima, contrarily to strong systems $(D \geq 15)$ which present lower density. As well, the so-called fragility index $m$ permits an assessment of the deviation of $\tau(T)$ from the Arrhenius behavior of polymers. It varies between two limiting values of 16 and $\geq 200$ for strong and fragile glass-formers, respectively [25], and can be obtained by Equation 9 .

$$
m=\left.\frac{d \log (\tau)}{d\left(T_{G} / T\right)}\right|_{T=T G}=\frac{B \cdot T}{\ln (10) \cdot\left(T_{G}-T_{V F T H}\right)^{2}} \mid
$$

\section{3. -Gel permeation chromatography}

RPLA-i samples were dissolved in chloroform (Fluka, purity of 99\%) at a concentration of $3-5 \mathrm{mg} / \mathrm{ml}$ at room temperature for 1 hand filtered for removal of contaminants before injecting the sample into the GPCcolumn. The polymers were analysed with a Verotech PL-GPC 50 Plus system equipped with a PL-RI Detector and two PLgel $5 \mu \mathrm{m}$ MIXED-D (300x7.5 mm) columns from Varian. The samples were injected with a PLAS RT Autosampler for PL-GPC 50 Plus, in which chloroform was used as mobile 
phase $\left(1 \mathrm{ml} / \mathrm{min}, 30^{\circ} \mathrm{C}\right)$. The calibration was created using polystyrene standards with a narrow molecular weight distribution. Corrections for the flow rate fluctuations were made using toluene as an internal standard. 


\section{Results and discussion}

\subsection{Dielectric response of virgin polylactide (VPLA)}

Figures 1, 2, 3 show the dielectric spectrum of the virgin polylactide VPLA in terms of the real part $\varepsilon^{\prime}$ (Fig 1), the imaginary part $\varepsilon^{\prime \prime}$ (Fig 2) of the dielectric permittivity, as well as $\operatorname{tg}(\delta)$ (Fig 3), respectively, at the frequency range from $10^{-2}$ to $10^{7} \mathrm{~Hz}$, for the isotherms in the temperature span of $0^{\circ} \mathrm{C} \leq T \leq 70^{\circ} \mathrm{C}$ (subplots a) and $75^{\circ} \mathrm{C} \leq T \leq 140^{\circ} \mathrm{C}$ (subplots c) . Different relaxation zones were observed at low and high temperatures.

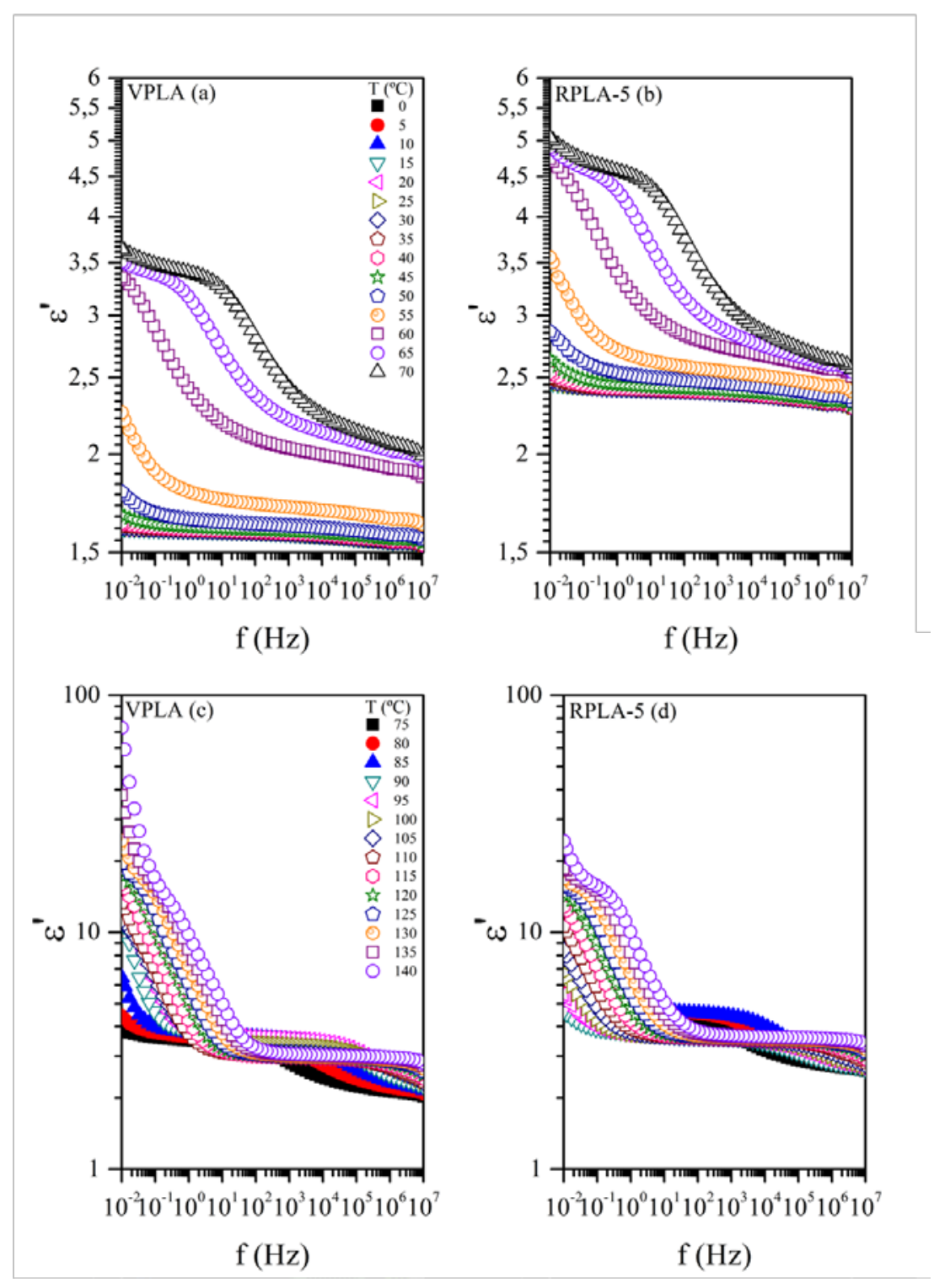

Figure 1. Isotherms showing the frequency response of virgin polylactide VPLA (left) and PLA reprocessed 5 times RPLA-5 (right) at temperatures range of $\left(0^{\circ} \mathrm{C} \leq T \leq 70^{\circ} \mathrm{C}\right)$ (up) and $\left(75^{\circ} \mathrm{C} \leq T \leq\right.$ $140^{\circ} \mathrm{C}$ ) (down) in terms of real component of the dielectric permittivity, $\varepsilon^{\prime}$. 

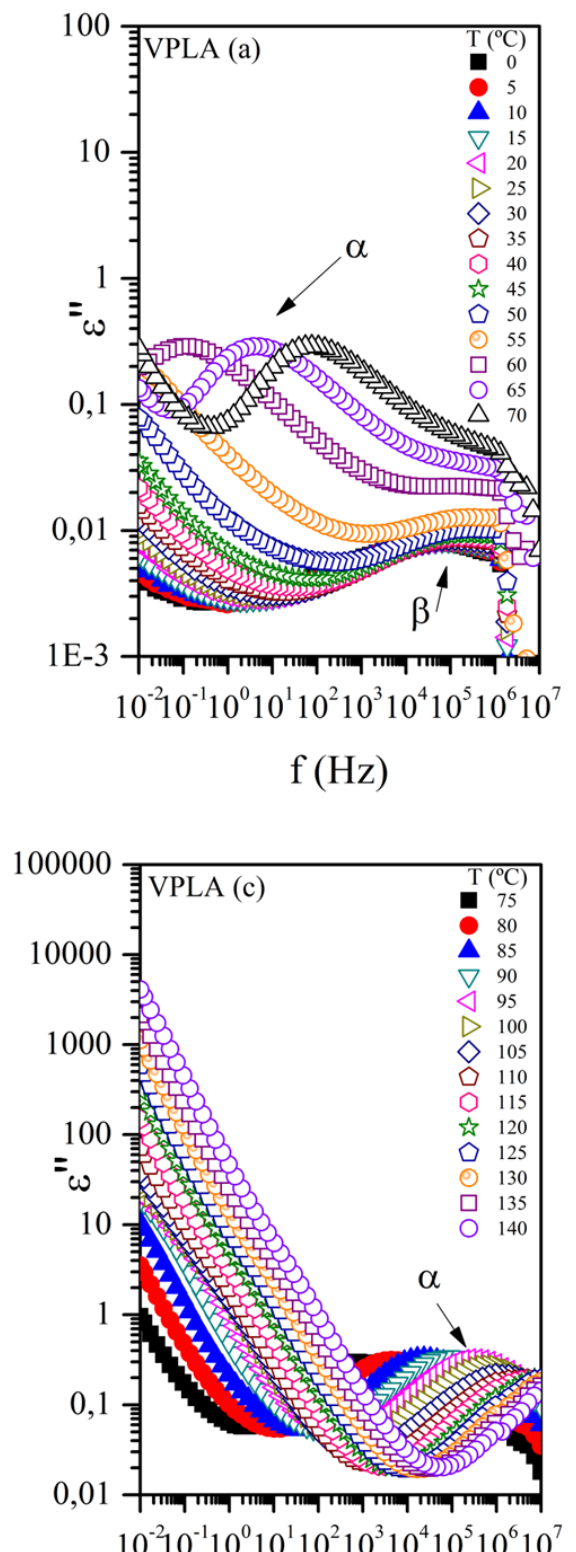

$\mathrm{f}(\mathrm{Hz})$
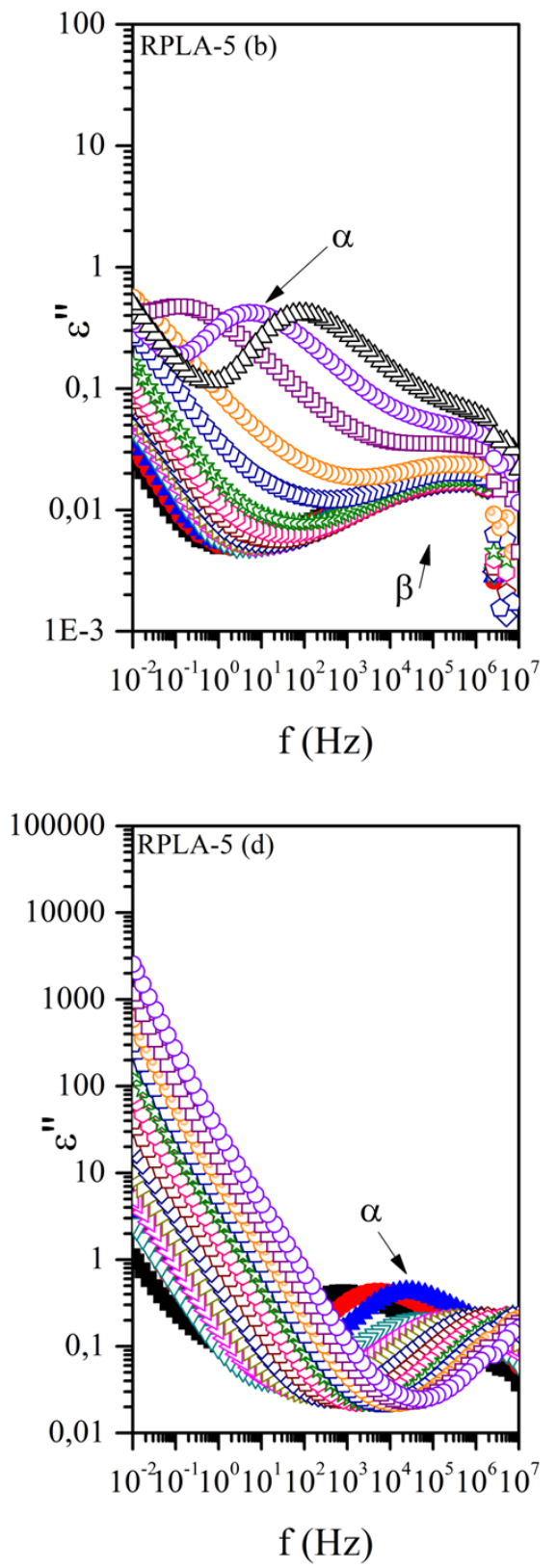

Figure 2. Isotherms showing the frequency response of virgin polylactide VPLA (left) and PLA reprocessed 5 times RPLA-5 (right) at temperatures range of $\left(0^{\circ} \mathrm{C} \leq T \leq 70^{\circ} \mathrm{C}\right)$ (up) and $\left(75^{\circ} \mathrm{C} \leq T \leq\right.$ $140^{\circ} \mathrm{C}$ ) (down) in terms of imaginary component of the dielectric permittivity, $\varepsilon^{\prime \prime}$. 

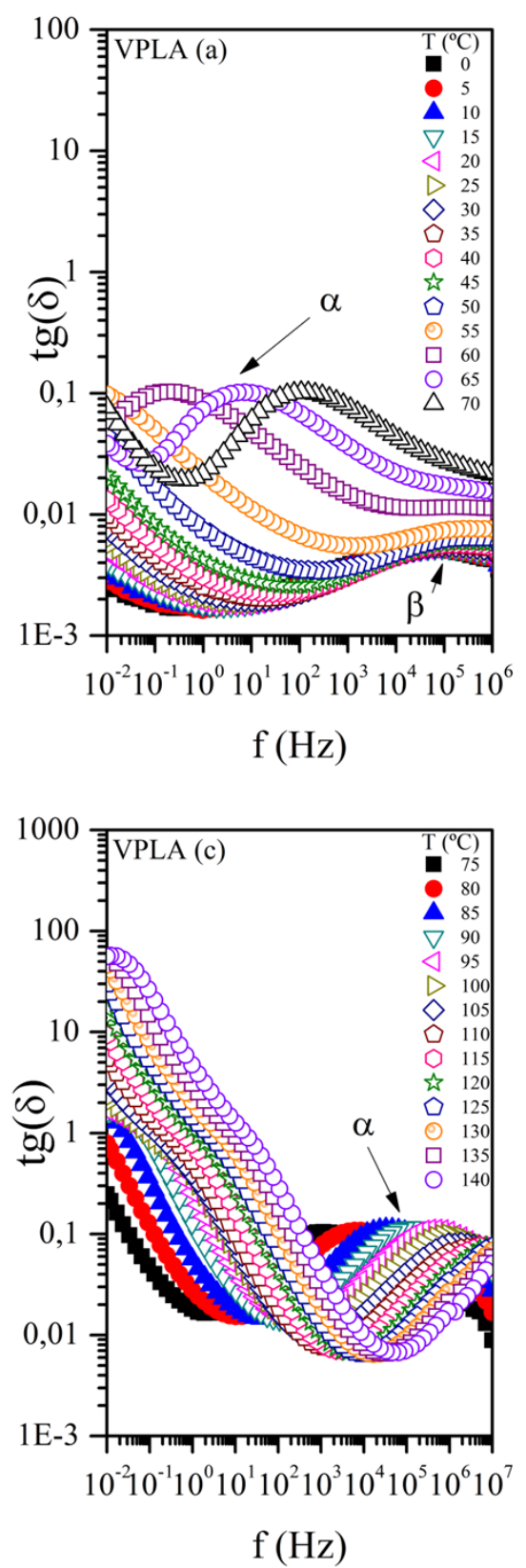
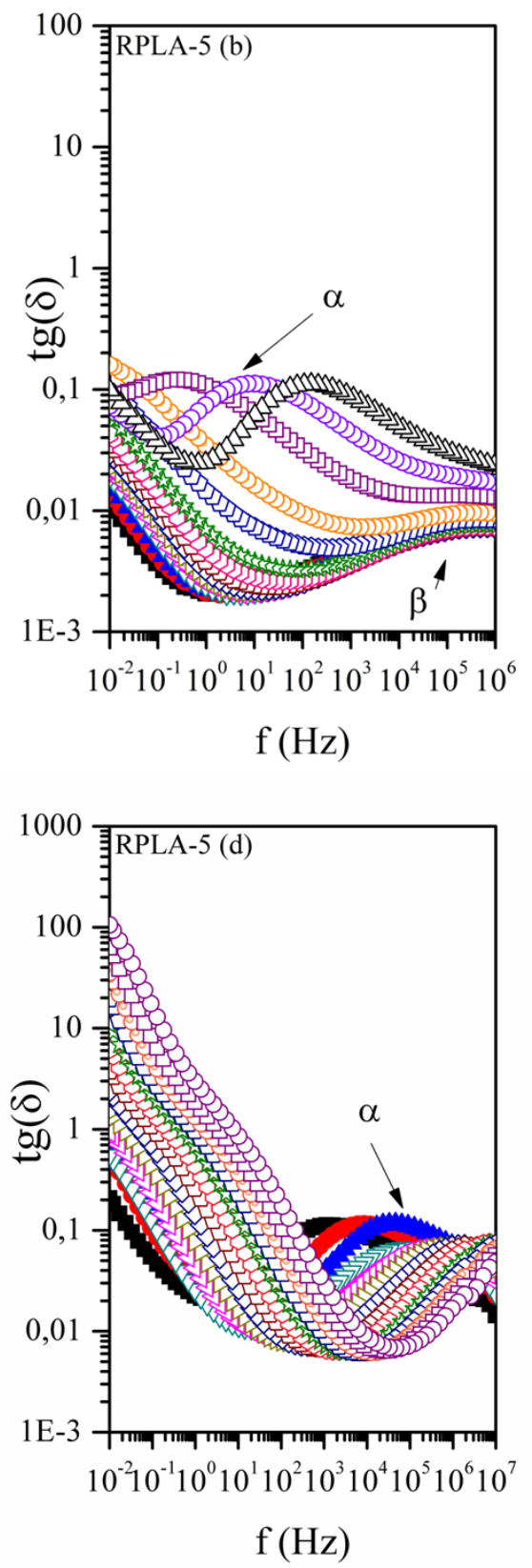

Figure 3. Isotherms showing the frequency response of virgin polylactide VPLA (left) and PLA reprocessed 5 times RPLA-5 (right) at temperatures range of $\left(0^{\circ} \mathrm{C} \leq T \leq 70^{\circ} \mathrm{C}\right)$ (up) and $\left(75^{\circ} \mathrm{C} \leq T \leq\right.$ $\left.140^{\circ} \mathrm{C}\right)($ down) in terms of loss tangent, $\operatorname{tg}(\delta)$.

A broad dielectric phenomenon, labelled as $\beta$ relaxation, was observed between $0^{\circ} \mathrm{C}$ and $50^{\circ} \mathrm{C}$ in a narrow range at high frequencies. As expected, the thermal activation of the $\beta$ dielectric relaxations followed a linear behaviour according to an Arrhenius temperature-dependence model (Eq.7), due to the non-cooperative nature of the local motions involved. The values of the apparent activation energy Ea related to this relaxation lied around $37-41 \mathrm{~kJ} \cdot \mathrm{mol}^{-1}$, in agreement with the bibliographic values, 
which confirmed that this relaxation process should be assigned to specific movements of terminal polar groups as carboxyl, hydroxyl and ester in the PLA branches as observed for other polymers. [22].

At higher temperatures, between $40^{\circ} \mathrm{C}$ and $120^{\circ} \mathrm{C}$, the $\alpha$ relaxation appeared. The thermal activation of this relaxation did not follow a linear behaviour according to the cooperative nature of the motions involved. Therefore, the results were fitted to VTFH model (Eq.8), as shown in Figure 4 with good linear regression coefficient, as it is given in Table 1. The $\alpha$ relaxation could be therefore assigned to movements of the segmental backbone, in accordance with previous experiments with DSC and DMTA [13]. Though the Tg obtained by DSC did not change with the reprocessing cycles, a deep inspection on the dielectric relaxations spectra at different frequencies can give information on the segmental rearrangements driven through the glass-rubber transition, in terms of dynamic fragility. It must be pointed out that at high temperatures and frequencies, Figure 4 shows a change in the shape of the VTFH curve, which shall be related to the rearrangement of chains during the cold-crystallization (at $T>T_{C C 0}$, i.e. the onset of the phenomenon) of PLA in the rubbery state [13], along the DETA experiment.

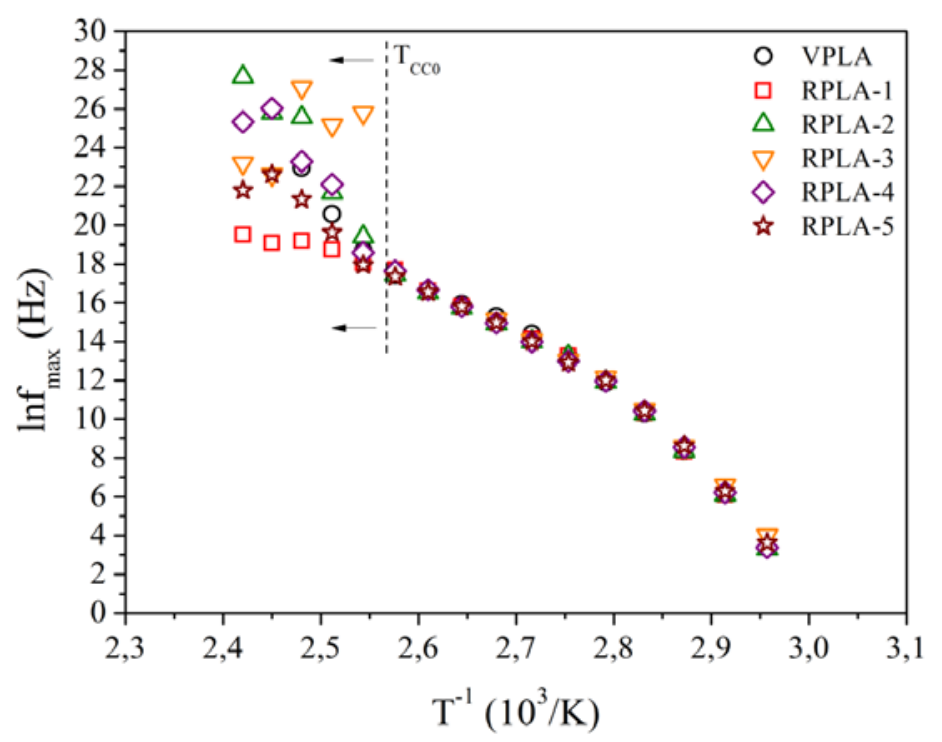

Figure 4. Arrhenius maps of the $\alpha$ relaxations corresponding to virgin and recycled polylactides (VPLA and RPLA-i). Deviation from VFTH model at temperatures higher than the cold-crystallization is remarked. 
Table 1. Results of fitting the thermal activation of the $\alpha$ dielectric relaxation to a VFTH model. Fragility parameters: $D, B, m$. Values of glass transition temperature and onset of cold crystallization obtained by differential scanning calorimetry are also referred [13].

\begin{tabular}{|c|c|c|c|c|c|c|c|c|}
\hline \multirow{2}{*}{ Material } & \multicolumn{2}{|c|}{ DSC } & \multicolumn{3}{c|}{ VFTH } & \multicolumn{3}{c|}{ Dynamic Fragility } \\
\cline { 2 - 9 } & $\mathrm{Tg}\left({ }^{\circ} \mathrm{C}\right)$ & $\mathrm{T}_{\mathrm{CC} 0}\left({ }^{\circ} \mathrm{C}\right)$ & $\mathrm{R}^{2}$ & $\mathrm{~T}_{\mathrm{VFTH}}\left({ }^{\circ} \mathrm{C}\right)$ & $\mathrm{D}$ & $\mathrm{B}(\mathrm{K})$ & $\mathrm{m}$ & $\Phi(\%)$ \\
\hline VPLA & $57,2 \pm 0,1$ & $106,2 \pm 1,7$ & 0,9990 & $15,8 \pm 0,4$ & $4,82 \pm 0,06$ & 1393 & 116 & 2,97 \\
\hline RPLA-1 & $56,7 \pm 0,1$ & $105,6 \pm 0,3$ & 0,9982 & $19,3 \pm 2,1$ & $4,23 \pm 0,06$ & 1239 & 126 & 3,03 \\
\hline RPLA-2 & $56,5 \pm 0,2$ & $102,0 \pm 0,1$ & 0,9982 & $22,0 \pm 2,1$ & $3,81 \pm 0,06$ & 1125 & 135 & 3,07 \\
\hline RPLA-3 & $56,7 \pm 0,3$ & $101,2 \pm 0,6$ & 0,9987 & $25,1 \pm 0,9$ & $3,18 \pm 0,04$ & 951 & 136 & 3,32 \\
\hline RPLA-4 & $56,8 \pm 0,1$ & $100,1 \pm 0,3$ & 0,9989 & $26,0 \pm 1,7$ & $3,18 \pm 0,04$ & 953 & 144 & 3,23 \\
\hline RPLA-5 & $56,6 \pm 0,1$ & $99,6 \pm 0,2$ & 0,9988 & $26,1 \pm 2,0$ & $3,12 \pm 0,04$ & 935 & 144 & 3,26 \\
\hline
\end{tabular}

\subsection{Dielectric response of recycled polylactides RPLA-i}

Figures 1, 2 and 3 show the dielectric spectrum of the polylactide reprocessed 5 times (RPLA-5) in terms of the real part $\varepsilon^{\prime}$ (Fig 1), the imaginary part $\varepsilon$ '” (Fig 2) of the dielectric permittivity, as well as $\operatorname{tg}(\delta)$ (Fig 3), respectively, at the frequency range from $10^{-2}$ to $10^{7} \mathrm{~Hz}$, for the isotherms in the temperature span of $0^{\circ} \mathrm{C} \leq T \leq 70^{\circ} \mathrm{C}$ (subplots b) and $75^{\circ} \mathrm{C} \leq T \leq 140^{\circ} \mathrm{C}$ (subplots $\mathrm{d}$ ). Intermediate results for the other recycled polylactides RPLA-1-2-3-4 were obtained, which are not presented for the sake of conciseness.

Concerning the evolution of $\varepsilon^{\prime}$, a general increase along the reprocessing cycles was found mainly at temperatures below the glass transition. However, afterwards the initial $\varepsilon$ ' were lower and the dielectric strength reduced. This can be explained in terms of dynamic fragility, as shown below. With regards to the evolution of the $\varepsilon$ "' and $\operatorname{tg}(\delta)$, a general displacement to higher frequency values was given. Following the procedure described for the virgin polylactide, the relaxation times of the different segmental motions were obtained for the recycled polylactide, for all temperature/frequency conditions. Figure 4 shows the Arrhenius maps for $\alpha$ relaxation. The thermal activation of both relaxations of all recycled polylactides RPLA-i followed the same behaviour as 
VPLA, being approachable by a VFTH model. Like VPLA, at high frequencies and temperatures, all recycled polylactides showed changes in the shape of the VTFH model, assigned to the cold-crystallization induced during the dielectric experiments. Indeed, the formation of more crystalline domains during the dielectric experiment was indicative of a higher presence of shorter polymer chains due to thermo-mechanical degradation the more the reprocessing cycles were applied [13].

Although in appearance the differences in thermal activation in Figure $\mathbf{4}$ could be small, a deeper look into the results of VFTH parameters can be helpful. The values corresponding to the fitting of the VFTH model are thus gathered in Table 1, with $R^{2}$ coefficients higher than 0.99. VPLA showed fragile glass-former performance, in agreement with other studies performed on fully amorphous PLA [26]. The influence of the structure of polymers on their fragility can be found elsewhere [27]. The dynamic fragility of the recyclates was higher the more the steps of mechanical recycling were applied, implying more chain cooperativity, thus explaining the reduction in dielectric strength previously found. The changes can be ascribed to an increase of mobility of polymer segments, due to more availability of shorter chains, due to thermo-mechanical degradation. The free volume coefficient $\Phi$ obtained by means of Eq (10) was also calculated to picture the change in cooperative movement due to thermo-mechanical degradation, and it is shown in Table 1.

$$
\phi=\frac{\left(T-T_{\mathrm{VFTH}}\right)}{B}
$$

Up to the second recyclate, the free volume showed an increase up to a $\sim 3,30 \%$ for the third and subsequent recyclates, showing a sort of threshold between both group of materials, i.e. VPLA, RPLA-1-2 on one side and RPLA-3-4-5 on the other side. This discussion is supported by the correlation of the dynamic fragility parameter $B$ in Table 1 with the vicous molar mass of the recyclates [13], which can be modelled by an exponential equation, as shown in Figure 5, with acceptable $R^{2}$ values, as shown in Table 2. In fact, after 3 reprocessing cycles, a reduction of a $\sim 25 \%$ in viscous molar mass resulted in a reduction of $\sim 31 \%$ of $B$. However, after that, a reduction of $\sim 21 \%$ in $M_{V}$ from RPLA-3 to RPLA-4 and $13 \%$ from RPLA-4 to RPLA-5, it scarcely ( 2\%) provoked a reduction of $B$. 


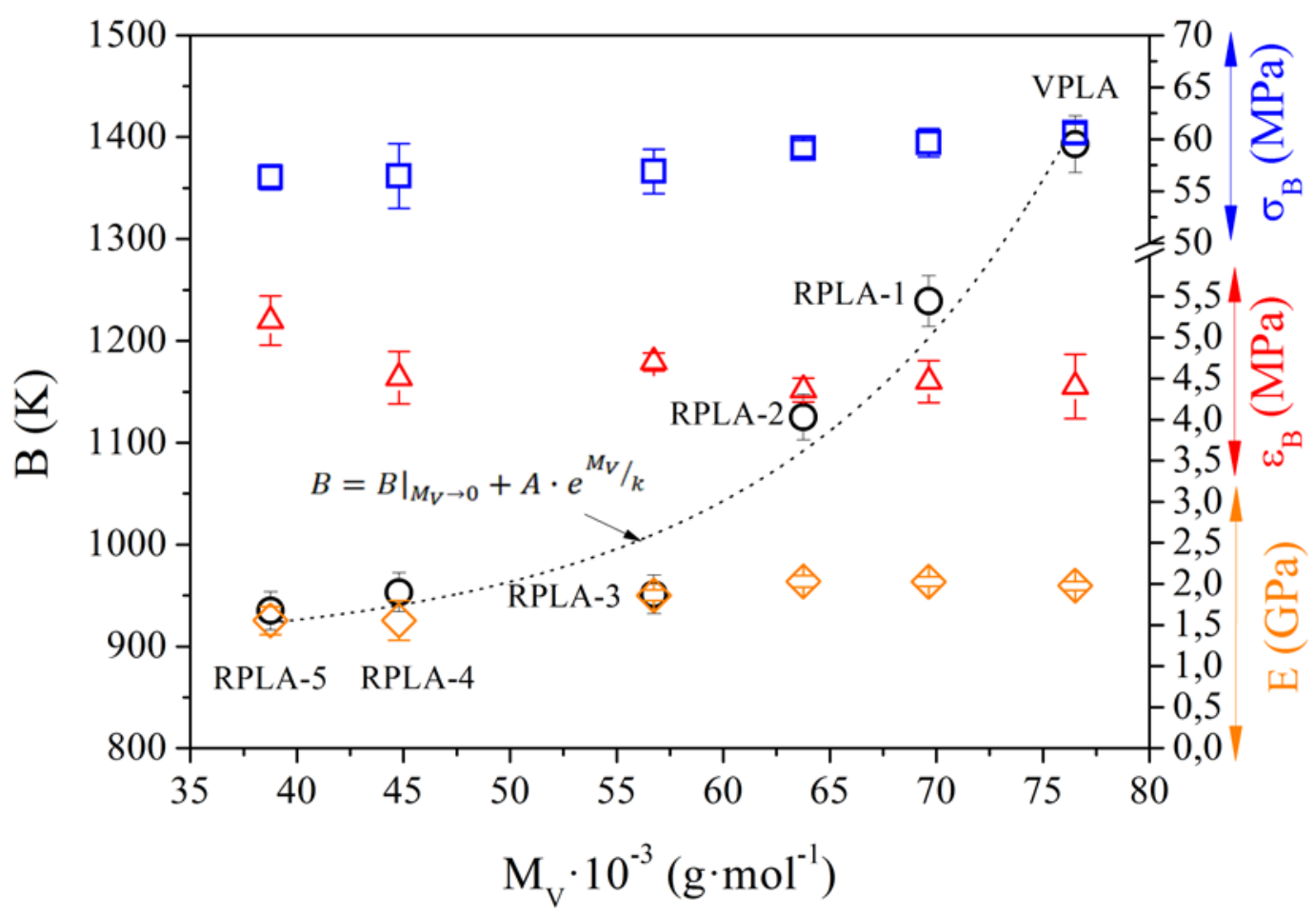

Figure 5. Exponential correlation of the fragility parameter $B$ with the decay of molar mass due to thermo-mechanical degradation. Comparison with macromolecular mechanical properties: stress at break $\sigma_{B}$, strain at break $\varepsilon_{B}$ and elastic modulus $E$.

Table 2. Results of fitting the relationship of molar mass and fragility parameter $B$ to exponential model

\begin{tabular}{|c|c|c|c|c|}
\hline \multirow{2}{*}{ Parameter } & Regression coefficient & Asymptotic minimum B & Amplitude of exponential & Growing parameter \\
\cline { 2 - 5 } & $\mathrm{R}^{2}$ & $\left.\mathrm{~B}\right|_{\mathrm{MV}_{\rightarrow}}(\mathrm{K})$ & $\mathrm{A}$ & $\mathrm{k}$ \\
\hline Value & 0,964 & $880,37 \pm 26,07$ & $2,858 \pm 0,27$ & $14670,43 \pm 156,08$ \\
\hline
\end{tabular}

GPC analyses were performed in this study to support the discussion on reductions of molar mass and widening of molar mass distributions, which are shown in Figure 6. Dynamic fragility is highly sensitive to macromolecular changes, in contrast to macroscopic mechanical properties, which were previously reported [13] and have been compared in this study, and the glass transition temperature obtained by DSC, given in Table 1. Thus, the application of Dielectric Spectroscopy can be of high interest to 
ascertain the quality of the material, by focusing on a molecular-level scenario. Parameters such as the stress at break $\sigma_{B}$, the strain at break $\varepsilon_{B}$ and the elastic modulus $E$ showed a change in tendency from RPLA-2 to RPLA-3. The evolution of these parameters is in agreement with previous results [13] in which thermal and chemical properties showed a change in PLA performance after the application of the second reprocessing step, obtaining limiting values regardless the number of processing cycles, thus suggesting a sort of threshold to be recovered by further mechanical recycling.

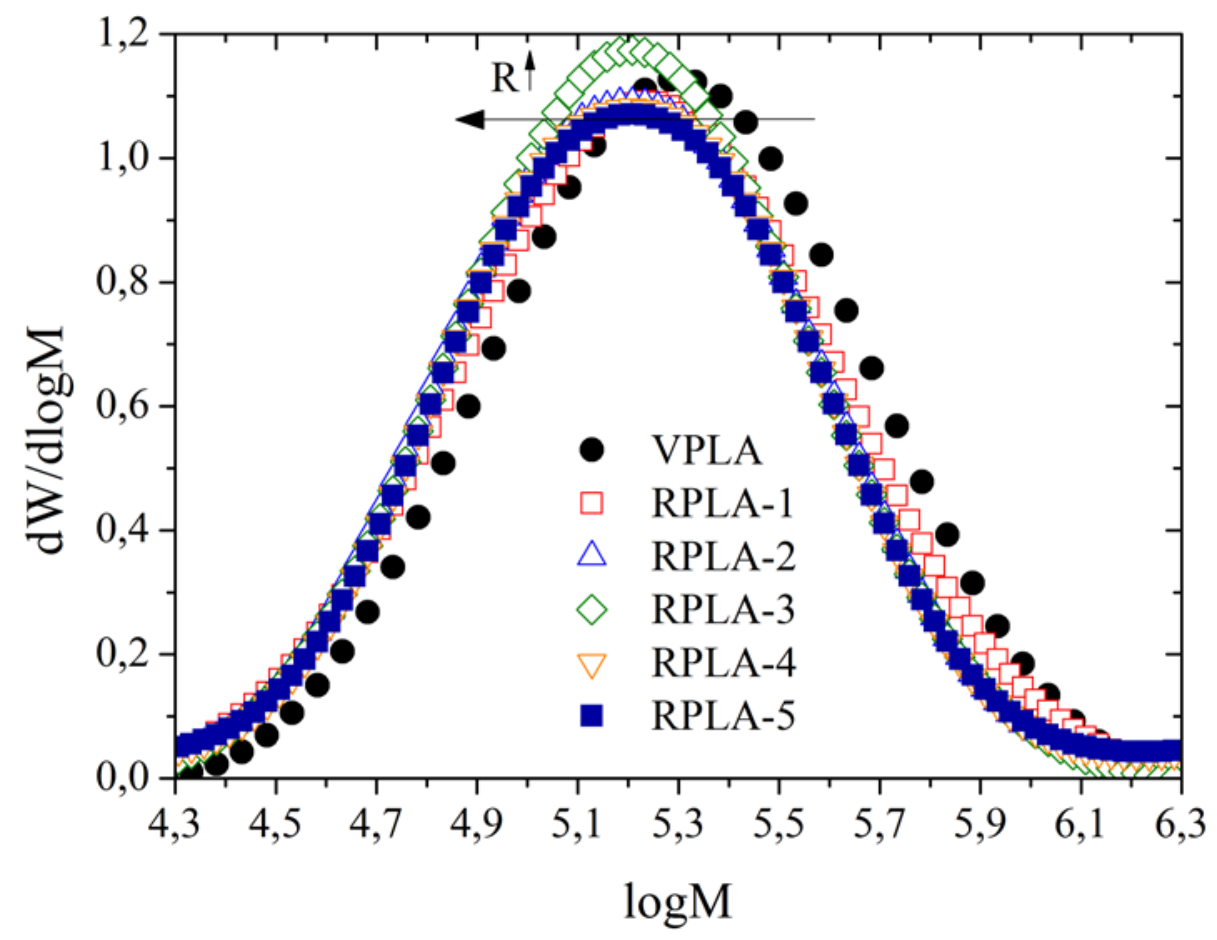

Figure 6. Molar mass distributions of recycled polylactide 


\section{Conclusions}

Dielectric spectroscopy measurements in a wide frequency/temperature range was used to analyse the influence of thermo-mechanical degradation induced by amorphous polylactide (PLA) submitted to in-plant recycling simulation up to 5 reprocessing cycles. Two relaxations zones $\alpha$ and $\beta$ in decreasing temperature order were found for all virgin and reprocessed materials, along with the apparition of the cold-crystallization at high temperatures and frequencies, favoured by the presence of shorter PLA segments due to thermo-mechanical degradation. The rearrangement of scissored chains into the amorphous matrix offered more free volume favouring the mobility of chains throughout the glass-rubber relaxation. This in turn provoked a reduction in the dynamic fragility of reprocessed PLA, exponentially correlated to the reduction of molar mass, particularly remarkable after the second reprocessing cycle.

\section{Acknowledgements}

The authors would like to acknowledge the Spanish Ministry of Economy and Competitiveness, through the Research Project ENE2011-28735-C02-01. The Vice-Rectorate for Research, Innovation and Technology Transfer of Universitat Politècnica de València is also thanked for additional support through PAID-06-SP20120581 project. 


\section{References}

1. Mohanty AK, Misra M, Hinrichsen G. Biofibres, biodegradable polymers and biocomposites: an overview. Macromolecular Materials and Engineering 2000;276:124.

2. Tsuji H, Doi Y. Biopolymers. Polyesters III. Applications and commercial products. In: Steinbüchel A, editor. Biopolymers. Weinheim: Wiley-VCH Verlag GMbh; 2002.

3. Auras R, Harte S, Selke S. An overview of polylactides as packaging materials. Macromolecular Bioscience 2004; 4:835-65.

4. Al-Salem SM, Lettieri P, Baeyens J. Recycling and recovery routes of plastic solid waste (PSW): a review. Waste Management 2009;29:2625-43.

5. Badia JD, Strömberg E, Ribes-Greus A, Karlsson S. A statistical design of experiments for optimizing the MALDI-TOF-MS sample preparation of polymers. an application in the assessment of the thermo-mechanical degradation mechanisms of poly (ethylene terephthalate). Analytica Chimica Acta 2011;692(1-2):85-95.

6. Badia JD, Strömberg E, Karlsson S, Ribes-Greus A. The role of crystalline, mobile amorphous and rigid amorphous fractions in the performance of recycled poly (ethylene terephthalate) (PET). Polymer Degradation \& Stability 2012;97(1):98-107.

7. Badia JD, Vilaplana F, Karlsson S, Ribes-Greus A. Thermal analysis as a quality tool for assessing the influence of thermo-mechanical degradation on recycled poly(ethylene terephthalate). Polymer Testing 2009;28(2):169-75.

8. Vilaplana F, Karlsson S. Quality concepts for the improved use of recycled polymeric materials: a review. Macromolecular Materials and Engineering 2008;293:274-97. 
9. Strömberg E, Karlsson S. The design of a test protocol to model the degradation of polyolefins during recycling and service life. Journal of Applied Polymer Science 2009;112:1835-44.

10. Vilaplana F, Ribes-Greus A, Karlsson S. Degradation of recycled high-impact polystyrene. Simulation by reprocessing and thermo-oxidation. Polymer Degradation and Stability 2006;91:2163-70.

11. Vilaplana F, Karlsson S, Ribes-Greus A. Changes in the micro-structure and morphology of high-impact polystyrene subjected to multiple processing and thermooxidative degradation. European Polymer Journal 2007;43:4371-81.

12. Badia JD, Strömberg E, Ribes-Greus A, Karlsson S. Assessing the MALDI-TOF MS sample preparation procedure to analyze the influence of thermo-oxidative ageing and thermo-mechanical degradation on poly (lactide). European Polymer Journal 2011;47(7):1416-28.

13. Badia JD, Strömberg E, Karlsson S, Ribes-Greus A. Material valorisation of amorphous polylactide. influence of thermo-mechanical degradation on the morphology, segmental dynamics, thermal and mechanical performance. Polymer Degradation and Stability 2012;97(4):670-8.

14. Badia JD, Santonja-Blasco L, Martínez-Felipe A, Ribes-Greus A. Hygrothermal ageing of reprocessed polylactide. Polymer Degradation and Stability 2012;97(10):1881-90.

15. Badia JD, Santonja-Blasco L, Martínez-Felipe A, Ribes-Greus A. Reprocessed polylactide: Studies of thermo-oxidative decomposition. Bioresource Technology 2012;114:622-8.

16. Badia JD, Santonja-Blasco L, Martínez-Felipe A, Ribes-Greus A. A methodology to assess the energetic valorization of bio-based polymers from the packaging industry: Pyrolysis of reprocessed polylactide. Bioresource Technology 2012;111:468-75. 
17. Santonja-Blasco L, Moriana R, Badía JD, Ribes-Greus A. Thermal analysis applied to the characterization of degradation in soil of polylactide: I. calorimetric and viscoelastic analyses. Polymer Degradation and Stability 2010;95(11):2192-9

18. Brottcher CJF, Borderwijk P. In: Theory of electric polarization, 2nd ed.; Elsevier: Amsterdam, 1978; Vol. 2, p. 72

19. Havriliak S, Negami S. In: A complex plane representation of dielectric and mechanical relaxation processes in some polymers. Polymer 1967,8 , 161

20. Havriliak S, Negami S. In: Dielectric and Mechanical Relaxation in Materials; Hanser: Munich, 1997

21. Ngai KL, Schonhals A, Schlosser E. An Explanation of Anomalous Dielectric Relaxation Properties of Polypropylene Glycol. Macromolecules 1992 , 25 , 4915

22. Santangelo P G, Roland CM. Molecular Weight Dependence of Fragility in Polystyrene Macromolecules 1998 , 31 , 4581

23. Vogel H. The Temperature Dependence Law of the Viscosity of Fluids. Phys. Z. $1921,22,645$

24. Fulcher G S. Analysis of Recent Measurements of the Viscosity of Glasses J. Am. Ceram. Soc. 1992 , 75 , 1043

25. Zuza E, Ugartemendia JM, López A, Meaurio E, Lejardi A, Sarasua JR. Glass transition behavior and dynamic fragility in polylactides containing mobile and rigid amorphous fractions. Polymer 2008; 49: 4427-4432

26. Mijovic J, Sy JW. Molecular dynamics during crystallization of poly(l-lactic acid) as studied by broad-band dielectric relaxation spectroscopy. Macromolecules 2002; 35 : 6370-6376 
27. Kunal K, Robertson CG, Pawlus S, Hahn SF, Sokolov AP. Role of chemical structure in fragility of polymers: a qualitative picture. Macromolecules 2008; 41: 7232-7238 
J. D. Badia, L. Monreal, V. Sáenz de Juano-Arbona A. Ribes-Greus. Dielectric spectroscopy of recycled polylactide. Polymer Degradation and Stability 2014; 107, 21-27. ISSN 0141-3910,

http://dx.doi.org/10.1016/j.polymdegradstab.2014.04.023.

\section{ANNEX. OPEN-ACCESS POLICIES}

One journal found when searched for: 0141-3910

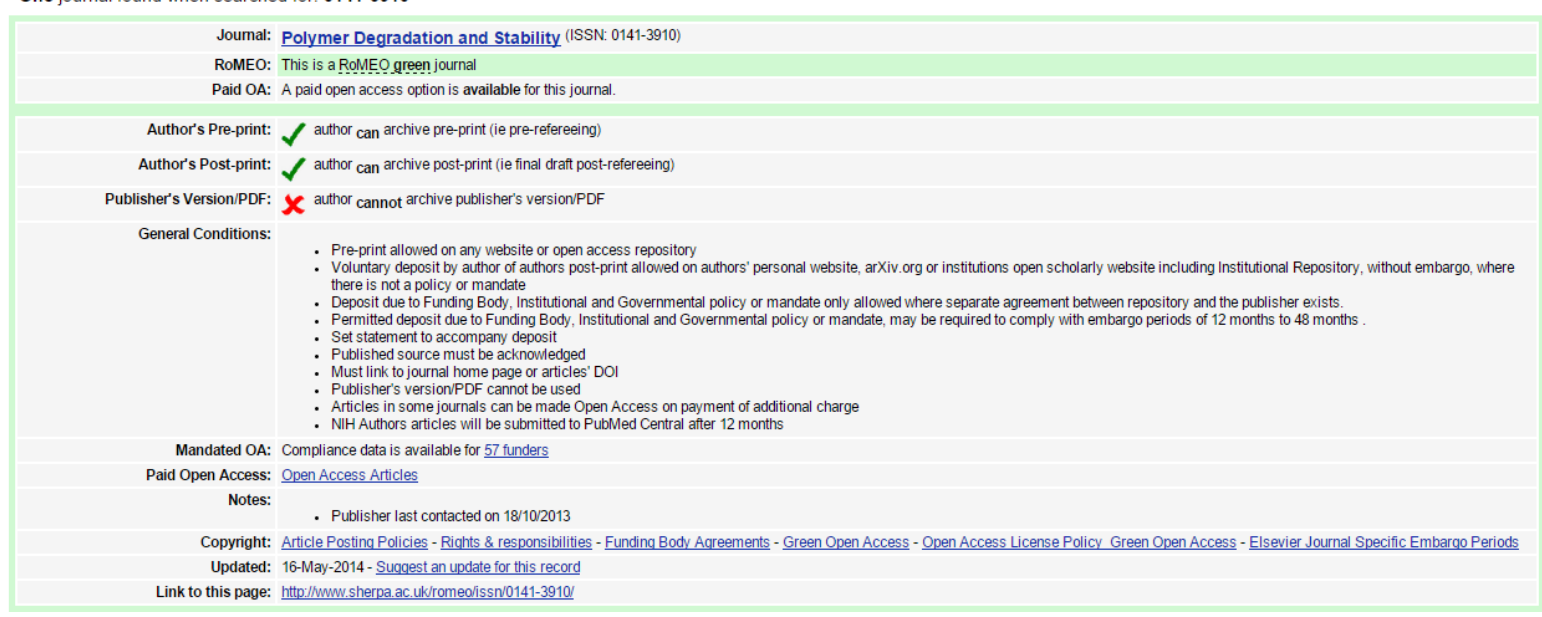

